\title{
Let your fingers do the walking: Finger force distinguishes competing accounts of the congruency sequence effect
}

\author{
Daniel H. Weissman ${ }^{1}$ \\ Published online: 21 June 2019 \\ (C) The Psychonomic Society, Inc. 2019
}

\begin{abstract}
The ability to remain flexible while engaging in goal-directed behavior is a critical aspect of cognitive control. A classic example of such flexibility is the congruency sequence effect (CSE) in distractor-interference tasks. In such tasks, participants respond more slowly when a distractor cues a different response than a target (incongruent trials) than when it cues the same response (congruent trials). However, this congruency effect is smaller after incongruent trials than after congruent trials. Interestingly, recent data from the prime-probe task indicate that this CSE is largest when participants treat the distractor, or prime, as the first of two temporally separated targets. It remains unclear, however, whether the CSE in this task indexes (1) control processes that modulate response activation before the second target (i.e., probe) appears or (2) congruency switch costs that delay responses after the probe appears. To distinguish between these accounts, participants' responses were recorded with force-sensitive keys. After responding to the prime, but before the probe appeared, participants exerted greater force on (1) the key cued by the prime after congruent trials, but on (2) the opposite key after incongruent trials. These findings indicate that the ability to remain flexible while engaging in goal-directed behavior relies on control processes that modulate response activation prior to the appearance of a goal-relevant stimulus.
\end{abstract}

Keywords Cognitive control $\cdot$ Conflict adaptation $\cdot$ Response force

\section{Introduction}

Remaining flexible while engaging in goal-directed behavior is crucial for survival. For example, a dog lover may decide to pet a stranger's dog after seeing the dog wag its tail, but not after hearing the dog growl. Such flexibility is a critical aspect of cognitive control, which allows humans to act in goal-driven, rather than stimulus-driven, ways (Miller \& Cohen, 2001).

\section{The congruency sequence effect (CSE)}

A prominent example of such flexibility appears in distractorinterference tasks. In such tasks, participants usually respond more slowly when a distractor cues a different response than a

Electronic supplementary material The online version of this article (https://doi.org/10.3758/s13423-019-01626-5) contains supplementary material, which is available to authorized users.

Daniel H. Weissman

danweiss@umich.edu

1 Department of Psychology, University of Michigan, 530 Church Street, Ann Arbor, MI 48109, USA target (incongruent trials) than when it cues the same response (congruent trials). However, this congruency effect is smaller after incongruent trials than after congruent trials (Gratton, Coles, \& Donchin, 1992). Researchers posit that this congruency sequence effect (CSE) indexes control processes that flexibly change the weight participants give to distractor information while identifying the target (Botvinick, Braver, Barch, Carter, \& Cohen, 2001; Gratton et al., 1992; Ridderinkhof, 2002).

Competing accounts vary with regard to when control processes engender the CSE. The attentional shift account posits that control processes modulate attention to the distractor and/ or target dimension before the next trial begins (Botvinick et al., 2001; Gratton et al., 1992). The response-modulation account posits that control processes modulate (e.g., inhibit) the response cued by the distractor after the distractor appears but before the target response is executed (Ridderinkhof, 2002; Stürmer, Leuthold, Soetens, Schroter, \& Sommer, 2002), possibly due to expectations regarding the upcoming target response (Weissman, Colter, Grant, \& Bissett, 2017). Finally, the congruency switch account posits that control processes reconfigure the cognitive system to employ different encoding processes after the target appears (e.g., to encode 
two unique responses in an incongruent trial vs. one in a congruent trial), thereby delaying responses, when the congruency of the current trial differs from that of the previous trial (Schmidt \& De Houwer, 2011).

Recent findings are more consistent with the responsemodulation and congruency switch accounts than with the attentional shift account. For example, consider a variant of the prime-probe task wherein a long (1-s) inter-stimulus interval (ISI) separates the distractor (prime) and target (probe), thereby eliminating the overall congruency effect. Here, the CSE is associated with a positive congruency effect after congruent trials and a negative congruency effect after incongruent trials (Weissman, Egner, Hawks, \& Link, 2015). A positive congruency effect after congruent trials could reflect a shift of attention toward the prime, an enhancement of the response cued by the prime, or congruency switch costs that slow performance in incongruent trials. Conversely, a negative congruency effect after incongruent trials could reflect an enhancement of the response opposite to the one cued by the prime or congruency switch costs that slow performance in congruent trials. However, it could not reflect a shift of attention away from the prime, as such a shift could eliminate, but not reverse, the congruency effect (Weissman et al., 2015). Therefore, the findings above favor the response-modulation and congruency switch accounts over the attentional shift account.

Prior data also appear more consistent with the responsemodulation account than with the congruency switch account. For example, using electroencephalography (EEG) (Stürmer et al., 2002) and response force (Miller \& Rouast, 2016), researchers showed that, after incongruent trials, distractorrelated response activation is reduced before the target response is executed. However, these researchers employed tasks with feature integration confounds (i.e., unequal stimulus and response repetitions in different sequential trial types), which could alter response activation independent of control processes (Hommel, Proctor, \& Vu, 2004). Therefore, these data do not provide direct support for the response-modulation account.

\section{The present study}

The goal of the present study was to distinguish between the response-modulation and congruency switch accounts. To this end, I measured participants' responses with force-sensitive keys while they performed a confound-minimized variant of the prime-probe task with long ISIs described above. This variant, which requires responding to both the prime and the probe (Grant \& Weissman, 2019; Weissman et al., 2017), yields a very large CSE for probe responses, consistent with prior data indicating the CSE is maximal when participants employ the same task set in consecutive trials (Hazeltine, Lightman, Schwarb, \& Schumacher, 2011). This finding indicates that the control processes underlying the CSE contribute to cognition in ways that are broader than minimizing distraction from irrelevant stimuli. Most important, I reasoned that this task would maximize the recruitment of control processes and, hence, statistical power for observing any effects of those processes on force. Specifically, the responsemodulation account predicts changes in force that vary with previous-trial congruency just before the probe appears. In contrast, the congruency switch account predicts no such changes.

\section{Methods}

\section{Participants}

Usable data from 24 participants were sought based on power analyses conducted with G*Power 3.1.9.2 (Faul, Erdfelder, Lang, \& Buchner, 2007). These analyses employed an alpha level of 0.05 and the partial eta-squared values associated with (1) the CSE in response time (RT) from our prior study of the confound-minimized prime-probe task $\left(\eta_{p}^{2}=0.756\right)$ (Grant \& Weissman, 2019) and (2) the CSE in pre-target force from a prior study of the Simon task $\left(\eta_{p}^{2}=0.406\right)$ (Miller \& Rouast, 2016). The results indicated that 24 participants would provide over 99\% power for observing a CSE in RT and 96\% power for observing a CSE in pre-target (i.e., pre-probe) force.

Twenty-nine undergraduates from the University of Michigan's Psychology Department subject pool participated in the study. Data were excluded from three students who experienced a computer error, one who performed the task with less than $70 \%$ accuracy, and one who self-reported a neuropsychiatric disorder. None of the remaining 24 participants (nine male, 15 female; mean age 18.71 years; age range $18-20$ years; $S D=0.75$ years) reported any history of head trauma, uncorrected visual or hearing impairments, seizures, or neurological/neuropsychiatric disorders.

Participants received course credit for taking part in the study. The University of Michigan's Behavioral Sciences Institutional Review Board approved the study protocol.

\section{Experimental design}

The experimental design was identical to that of our prior study of the same task (Grant \& Weissman, 2019, Experiment 2). A fixation cross $\left(0.8^{\circ} \times 0.8^{\circ}\right)$ appeared for $2 \mathrm{~s}$ at the start and end of each block. Further, each 3-s trial consisted of four sequential events (Fig. 1a): (1) a large arrow (duration, $133 \mathrm{~ms}$ ), (2) a blank screen (duration, 1,000 ms), ${ }^{1}$ (3) a small arrow (duration, $133 \mathrm{~ms}$ ), and (4) a second blank

\footnotetext{
${ }^{1}$ See the Supplementary Material for Footnote 1
} 
a)

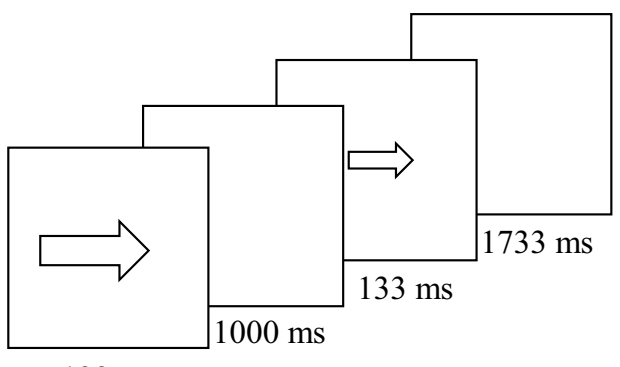

$133 \mathrm{~ms}$

b)

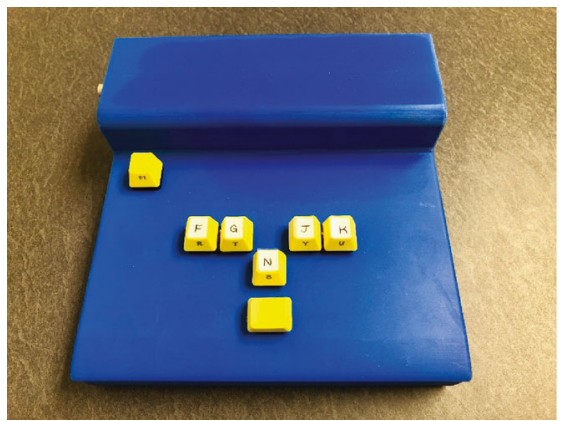

Fig. 1 The prime-probe task and custom response box. (A) In each trial, participants indicated the direction in which (1) the first arrow (or prime) pointed during the initial (1000 ms) ISI and (2) the second arrow (or probe) pointed during the subsequent $(1733 \mathrm{~ms})$ ISI. In congruent trials (top left), the arrows pointed in the same direction. In incongruent trials (top right), they pointed in opposite directions. (B) The custom response box with force-sensitive keys that I employed in the present study. Participants pressed the F, G, J, or N key to indicate whether each arrow

screen (duration, 1,733 ms). The two arrows in each trial were both horizontally-aligned (i.e., left and/or right arrows; prime, $7.8^{\circ} \times 4.7^{\circ}$; probe, $2.6^{\circ} \times 1.6^{\circ}$ ) or both vertically-aligned (i.e., up and/or down arrows; prime, $4.7^{\circ} \times 7.8^{\circ}$; probe, $1.6^{\circ} \times$ $2.6^{\circ}$ ). All stimuli appeared in white on a black background.

There were two trial types. In congruent trials (Fig. 1a, left), the large and small arrows pointed in the same direction. In incongruent trials (Fig. 1a, right), they pointed in opposite directions. There were four unique congruent pairs of large and small arrows (left-left, right-right, up-up, down-down) and four unique incongruent pairs (left-right, right-left, up-down, down-up).

Three constraints were imposed on the trial sequence for each block. First, to avoid stimulus and response repetition confounds in consecutive trials (Hommel et al., 2004), left and/or right arrows were presented in odd-numbered trials and up and/or down arrows were presented in even-numbered trials. Second, each unique congruent and incongruent arrow pair appeared equally often. Third, the trials were presented in a unique, pseudo-randomized order. This ensured that congruent and incongruent trials were each preceded by congruent and incongruent trials equally often, separately for odd and even trials. Each of the resulting four sequential-congruency trial

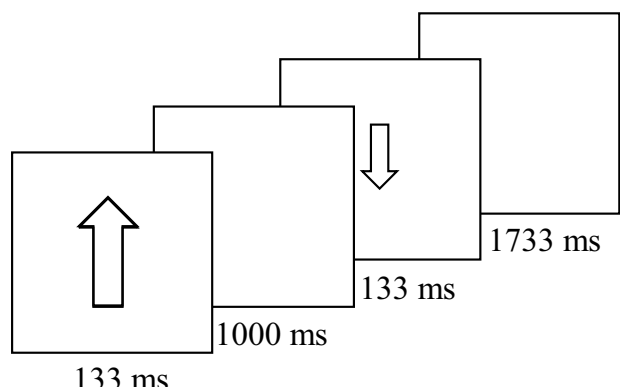

c)

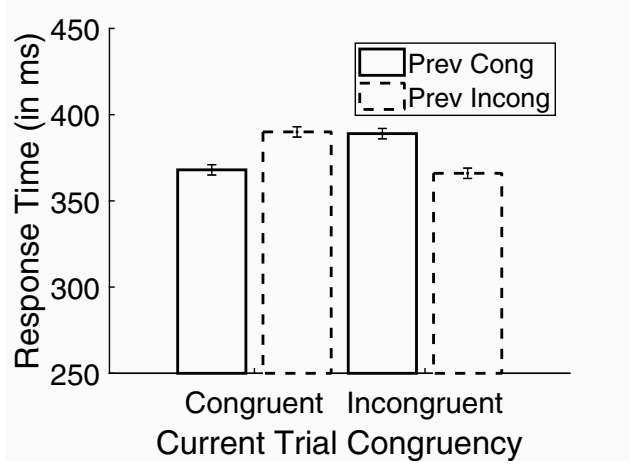

pointed left, right, up, or down, respectively. (C) The congruency sequence effect, or CSE, in mean response time (RT). Mean RT was faster when the congruency of the previous trial (congruent or incongruent) matched the congruency of the current trial (congruent or incongruent) than when it did not. Error bars indicate \pm 1 within-subject standard error of the mean (for more details, see https://motorbehaviour.wordpress.com/ 2014/10/31/confidence-intervals-in-within-participant-design-a-tutorialon-the-cousineau-morey-method/)

types appeared 16 times in each block, except for one that appeared only 15 times because no trial preceded the first trial.

\section{Apparatus}

A custom response box was employed that continuously measures analog response force and digital keypresses (Fig. 1b; see the Supplementary Material for a full description). The response box has five force-sensitive keys and two standard keys. The force-sensitive (F, G, J, K, and N) keys are spaced as on a QWERTY keyboard. Each key reliably detects analog changes in mass as small as $100 \mathrm{mg}$ at a sampling rate of 200 Hz. The standard keys (space bar and escape) appear at the bottom and top left corner of the box.

\section{Procedure}

A 64-trial practice block preceded ten 64-trial test blocks. Participants were instructed to respond as quickly and accurately as possible to each arrow using the force-sensitive keys. Specifically, they were instructed to respond to left arrows by pressing the " $\mathrm{F}$ " key (left middle finger), to right arrows by 
pressing the "G" key (left index finger), to up arrows by pressing the " $\mathrm{J}$ " key (right middle finger), and to down arrows by pressing the "N" key (right index finger). If a participant responded incorrectly or failed to respond within $900 \mathrm{~ms}$ of stimulus onset, the word "Error" appeared centrally for 200 ms. The Psychophysics Toolbox (Brainard, 1997) was employed on a Windows 10 PC to present the stimuli. The custom response box described earlier was employed to record analog and digital keypress data.

\section{Data analyses}

The mean RT and mean error rate (ER) data for probe responses were analyzed using separate repeated-measured analyses of variance (ANOVAs). The ANOVAs included two factors: (1) previous-trial congruency (congruent, incongruent) and (2) current-trial congruency (congruent, incongruent). In the analysis of mean RT, outliers (i.e., trials with RTs more than three standard deviations from their condition means), errors (involving either the prime response or the probe response), and trials after errors were excluded. In the analysis of mean ER, only outliers and trials after errors were excluded. On average, overall accuracy was high (90.2\%), and the percentage of outliers was low (1.2\%).

The force data were analyzed in two ways. First, in a stimuluslocked analysis, whether mean force $933-1,133 \mathrm{~ms}$ after prime onset (200-0 ms before probe onset) varied with previous-trial congruency on the prime-congruent key (the key cued by the prime arrow) and/or the prime-incongruent key (the key opposite to the one cued by the prime arrow) was examined. I analyzed the force data from $933-1,133 \mathrm{~ms}$ after prime onset because (1) participants had only $900 \mathrm{~ms}$ to respond to the prime and (2) mean RT was only about $400 \mathrm{~ms}$ in our prior study of the same task (Grant \& Weissman, 2019). For these reasons, it was judged that analyzing force from $933-1,133 \mathrm{~ms}$ after prime onset would most effectively distinguish (1) control processes that modulate response activation just prior to an upcoming probe from (2) motor processes that respond to the preceding prime. The force data were averaged across all of the time points in this 200-ms window, as it was thought that control processes would operate across the entire window, in which case averaging would increase the signal-to-noise ratio. Consistent with this approach, prior studies of both the congruency effect and the CSE have aggregated force data across an entire 200-ms window (Mattler, 2005; Miller \& Rouast, 2016).

Second, in a response-locked analysis, whether force effects similar to those described above appeared $515-715 \mathrm{~ms}$ after participants responded to the prime was examined. Each analysis employed a repeated-measures ANOVA with two factors: (1) previous-trial congruency (congruent, incongruent) and (2) response key (prime-congruent, prime-incongruent). The same trials as in the analysis of mean RT were excluded.

\section{Results}

\section{Mean RT}

There was an interaction between previous-trial congruency and current-trial congruency, $F(1,23)=119.895, p<0.001, \eta_{p}^{2}$ $=0.839$ (Fig. 1c). Replicating our prior findings from this task (Grant \& Weissman, 2019), there was a positive (21 ms) congruency effect after congruent trials, $F(1,23)=13.985, p=$ $0.001, \eta_{p}^{2}=0.378$, and a negative $(-24 \mathrm{~ms})$ congruency effect after incongruent trials, $F(1,23)=25.579, p<0.001, \eta_{p}^{2}=$ 0.527 . $^{2}$ No other effects were significant.

\section{Mean ER}

The interaction observed in the mean RT data and the two main effects were significant (see the Supplementary Materials).

\section{Stimulus-locked prime force}

Figure 2 shows the stimulus-locked prime force across time as a function of previous-trial congruency on (1) the primecongruent key and (2) the prime-incongruent key. Mean force 933-1,133 ms after prime onset was examined (200-0 ms before probe onset) (Fig. 2c).

There were two significant effects. First, there was a main effect of response key, $F(1,23)=8.713, p<0.007, \eta_{p}^{2}=0.275$ : participants exerted greater force on the prime-congruent key $(18.49 \mathrm{cN})$ than on the prime-incongruent key $(17.06 \mathrm{cN})$. Second, there was an interaction between response key and previous-trial congruency, $F(1,23)=17.508, p<0.001, \eta_{p}^{2}=$ 0.432 . Consistent with the response-modulation account, but not with the congruency switch account, participants exerted greater force on the prime-congruent key after congruent trials than after incongruent trials $(18.78 \mathrm{cN}$ vs. $18.21 \mathrm{cN}), F(1,23)$ $=10.240, p=0.004, \eta_{p}^{2}=0.308$. Further, they exerted greater force on the prime-incongruent key after incongruent trials than after congruent trials $(17.25 \mathrm{cN}$ vs. $16.88 \mathrm{cN}), F(1,23)$ $=8.893, p=0.007, \eta_{p}^{2}=0.279$. These findings suggest that participants prepared (1) the response cued by the prime after congruent trials and (2) the response opposite to the one cued by the prime after incongruent trials. No other effects were significant.

\section{Response-locked prime force}

Stimulus-locked prime force varied with previous-trial congruency soon after prime onset (Figs. 2a-b), likely because

\footnotetext{
${ }^{2}$ See the Supplementary Material for Footnote 2.
} 
a)

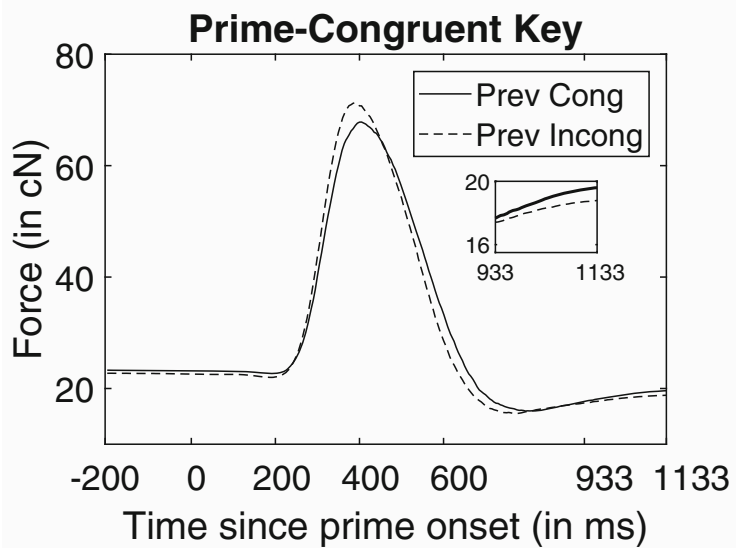

b)

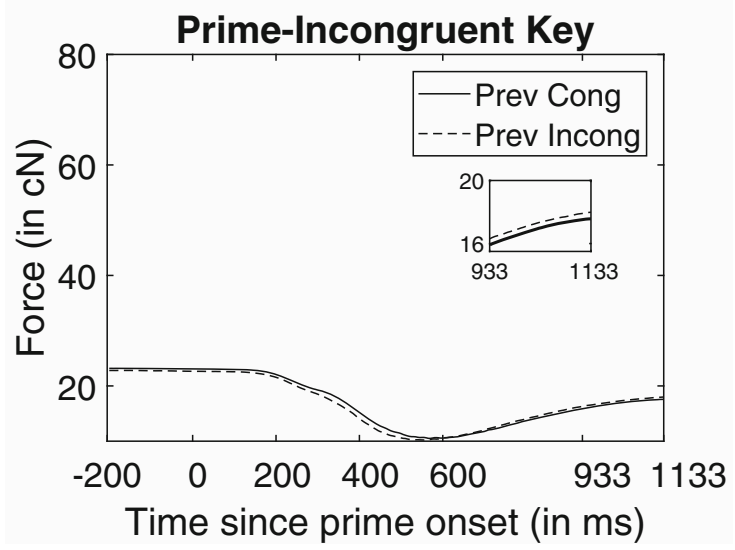

c)

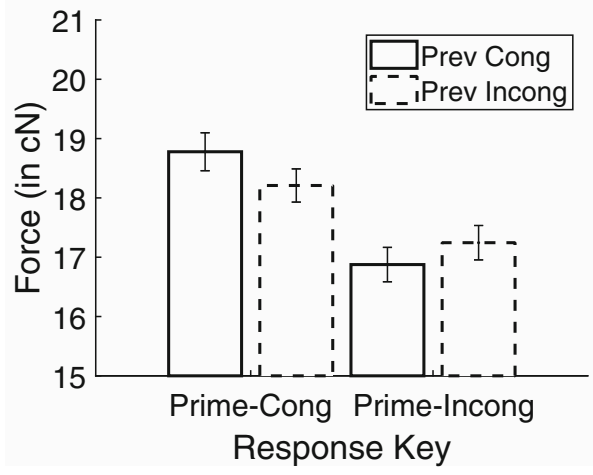

Fig. 2 Mean force across 24 participants time-locked to prime onset. (A) Mean force on the prime-congruent response key (i.e., the key participants pressed when the prime appeared) in centinewtons $(\mathrm{cN})$ as a function of previous-trial congruency (congruent, incongruent) and time since prime onset. The subfigure focuses on $933-1133 \mathrm{~ms}$ after prime onset. (B) Mean force on the prime-incongruent key (i.e., the key opposite to the one cued by the prime) in $\mathrm{cN}$ as a function of previous-trial congruency (congruent, incongruent) and time since prime onset. The subfigure focuses on 933-1133 ms after prime onset. (c) Mean force 933-1133 ms after prime onset as a function of response key (prime-congruent, primeincongruent) and previous-trial congruency (congruent, incongruent). After responding to the prime, but before the probe appeared, participants exerted greater force on the prime-congruent key after congruent trials but on the prime-incongruent key after incongruent trials. Error bars indicate \pm 1 within-subject standard error of the mean (https://motorbehaviour. wordpress.com/2014/10/31/confidence-intervals-in-within-participantdesign-a-tutorial-on-the-cousineau-morey-method/)

participants responded to the prime, because, on average, this corresponded to 929-1,129 ms after prime onset (similar to the stimulus-locked analysis). As the slowest participant's mean prime RT was just $80 \mathrm{~ms}$ longer than the group average, it is unlikely that probe-related force influenced this analysis.

There were three significant effects (Fig. 3c). First, there was a main effect of response key, $F(1,23)=8.075, p=0.009$, $\eta_{p}^{2}=0.260$ : participants exerted greater force on the primecongruent key $(18.739 \mathrm{cN})$ than on the prime-incongruent key $(17.427 \mathrm{cN})$. Second, there was a main effect of previous-trial congruency, $F(1,23)=6.619, p=0.017, \eta_{p}^{2}=0.223$ : participants exerted greater force after congruent trials $(18.22 \mathrm{cN})$ than after incongruent trials $(17.946 \mathrm{cN})$. Third, there was an interaction between response key and previous-trial congruency, $F(1,23)=23.578, p<0.001, \eta_{p}^{2}=0.506$. Consistent with the response-modulation account (and with the stimuluslocked results), but not with the congruency switch account,

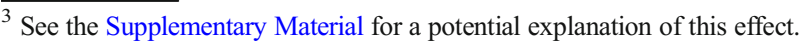


a)

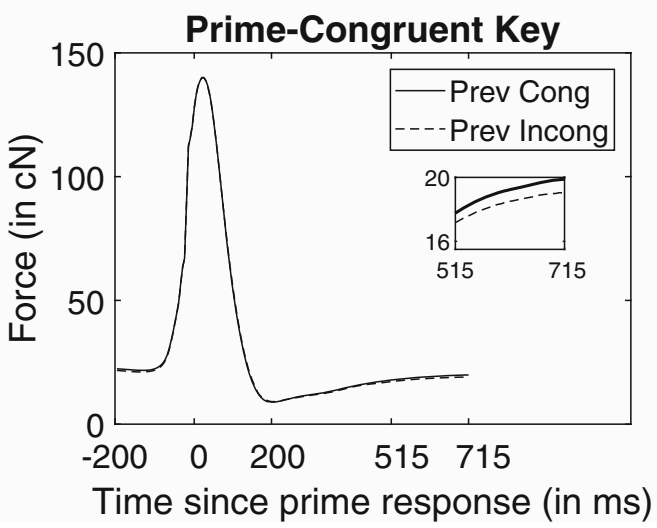

b)

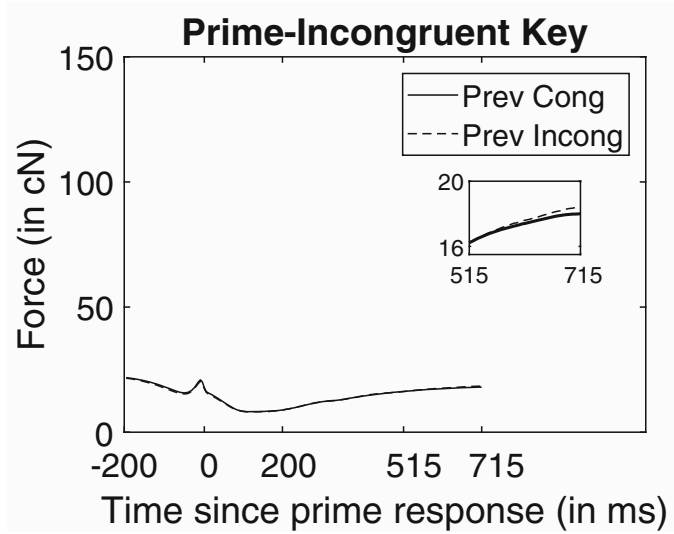

c)

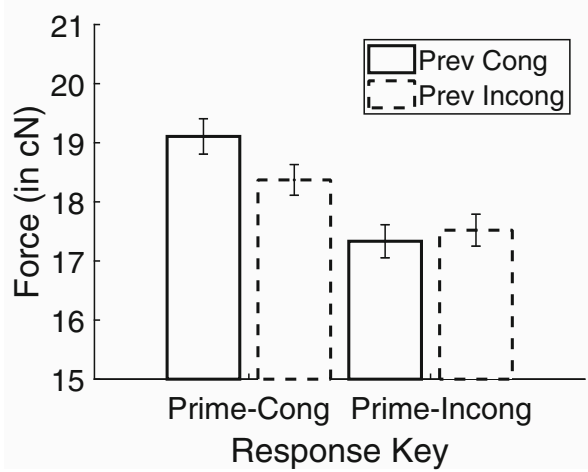

Fig. 3 Mean force across 24 participants time-locked to prime response onset. (A) Mean force on the prime-congruent response key (i.e., the key participants pressed when the prime appeared) in centinewtons $(\mathrm{cN})$ as a function of previous-trial congruency (congruent, incongruent) and time since prime response onset. The subfigure focuses on 515-715 ms after prime response onset. (B) Mean force on the prime-incongruent key (i.e., the key opposite to the one cued by the prime) in $\mathrm{cN}$ as a function of previous-trial congruency (congruent, incongruent) and time since prime response onset. The subfigure focuses on 515-175 $\mathrm{ms}$ after prime response onset. (c) Mean force 515-715 ms after prime

participants exerted greater force on the prime-congruent key after congruent trials than after incongruent trials $(19.11 \mathrm{cN}$ vs. $18.37 \mathrm{cN}), F(1,23)=21.627, p<0.001, \eta_{p}^{2}=0.485$. Further, they exerted greater force on the prime-incongruent key after incongruent trials than after congruent trials (17.52 $\mathrm{cN}$ vs. $17.33 \mathrm{cN})$, although this difference was not significant, $F(1,23)=2.219, p=0.15, \eta_{p}^{2}=0.088$. No other effects were significant.

\section{Exploratory analysis}

One possible interpretation of the present findings is that participants form different expectations about the upcoming probe response following congruent versus incongruent trials and modulate response activation accordingly. Specifically,

\footnotetext{
${ }^{0}$ Tyler Adkins suggested this possibility.
}

response onset as a function of response key (prime-congruent, primeincongruent) and previous-trial congruency (congruent, incongruent). After responding to the prime, but before the probe appeared, participants exerted greater force on the prime-congruent key after congruent trials but on the prime-incongruent key after incongruent trials (although the latter effect was not significant). Error bars indicate \pm 1 within-subject standard error of the mean (https://motorbehaviour.wordpress.com/2014/10/31/ confidence-intervals-in-within-participant-design-a-tutorial-on-thecousineau-morey-method/).

they expect the congruency of the current trial to match the congruency of the previous trial. Hence, after identifying the prime, they prepare an expected (congruent or incongruent) response to the probe. The present data do not allow a definitive test of this hypothesis. However, they invite an exploratory analysis of whether response expectations influence force. Specifically, as the odd and even trials in each block involved left- and right-hand responses, participants may have expected to use their left and right hands in odd and even trials, respectively, and prepared the relevant effectors. Consistent with this possibility, 200-0 ms before prime onset, participants exerted greater force on the two keys (e.g., F and G) they would use in the upcoming (e.g., odd) trial $(22.95 \mathrm{cN})$ than on the two keys (e.g., J and N) they used in the previous (e.g., even) trial (21.28 $\mathrm{cN}), F(1,23)=11.879, p=0.002, \eta_{p}^{2}=0.341$. These findings suggest that force measures could be useful for investigating whether response expectations engender the CSE. 


\section{Discussion}

The ability to adapt flexibly to changing conditions while engaging in goal-directed behavior is an important aspect of cognitive control. A classic example of such flexibility is the CSE. Here, I sought to distinguish between two accounts of the CSE in the prime-probe task. The response-modulation account posits the CSE indexes changes in response activation that vary with previous-trial congruency before the probe appears. The congruency switch account posits the CSE indexes different encoding processes after the probe appears. Critically, the present findings were more consistent with the response-modulation account than with the congruency switch account. Specifically, just before the probe appeared, participants exerted greater force on (1) the prime-congruent key after congruent trials but on (2) the prime-incongruent key after incongruent trials. These findings show that remaining flexible while engaging in goal-directed behavior relies on control processes that modulate response activation before a goal-relevant stimulus appears. This outcome has important implications for current accounts of the CSE.

\section{Implications for the response-modulation account}

The present findings provide direct support for the responsemodulation account. As such, they fit with our prior suggestion that the CSE indexes interactions between episodic memory and cognitive control (Weissman, Hawks, \& Egner, 2016). In this view, control processes underlying the CSE combine (1) a memory of previous-trial congruency (e.g., incongruent) with (2) the identity of the current-trial prime (e.g., up arrow) to predict (3) a response to the upcoming probe (e.g., down key) (Weissman et al., 2017).

One may wonder, though, why control processes should predict any response to the probe. Since trial congruency varies randomly across trials, such predictions cannot achieve more than chance accuracy. However, people often form expectations based on local sections of random sequences. In two alternative-forced-choice tasks, for example, they respond more quickly in trials wherein (1) the stimulus repeats after experiencing stimulus repetitions and (2) the stimulus alternates after experiencing stimulus alternations (Huettel, Mack, $\&$ McCarthy, 2002). This raises the possibility that control processes underlying the CSE predict the prime and probe will involve (1) the same stimulus and/or response if they did so in the previous trial or (2) different stimuli and/or responses if they did so in the previous trial, even when no predictive relationship exists (Egner, 2014). Consistent with this possibility, in unstable environments, the most recent event is often the best predictor of the current environmental state and, hence, the nature of the next event (Behrens, Woolrich, Walton, \& Rushworth, 2007). On the other hand, if there is a predictive relationship between previous- and current-trial congruency, control processes might override the "history repeats itself" heuristic, consistent with the present exploratory analysis. ${ }^{4}$ Future studies could investigate these possibilities.

\section{Implications for the congruency switch account}

The present findings challenge the congruency switch account, which does not predict changes in pre-probe force (Schmidt \& De Houwer, 2011). However, they do not rule out the possibility that congruency switch costs contribute to the CSE after the probe appears. Consistent with this possibility, the use of different encoding processes in consecutive trials, which is thought to engender congruency switch costs, contributes to switch costs in studies of task switching (Logan $\&$ Bundesen, 2003). Still, the present findings weigh against the possibility that congruency switch costs are the sole source of the CSE.

\section{Broader implications}

In the present study, custom response boxes equipped with force-sensitive keys that look and feel like those on a standard QWERTY keyboard were employed. Unlike mouse tracking, EEG, or functional MRI, this approach allows one to inexpensively measure multiple finger-specific response activations on the same hand with a high signal-to-noise ratio. Hence, this approach may also be useful for investigating other phenomena that engender systematic changes in response activation, such as decision-making, sequence learning, and motor control.

\section{Conclusion}

The present findings favor the response-modulation account of the CSE over the congruency switch account. They also highlight the usefulness of response force measures for distinguishing between competing accounts of cognitive control. Therefore, future studies that employ such measures may provide additional insights into flexible, goal-directed behavior.

Author Note The author thanks Andrea Dai, Cathryn Goldman, Lauren Grant, Ceren Ege, Al-Amin Ali, Alexis Salinas, and Daphne Samuel for assisting with data collection. The author also thanks Jeff Miller and Lauren Grant for useful discussions and Lauren Grant, Tyler Adkins, and John Plass for providing feedback on earlier drafts of this article. Finally, the author thanks Cameron Nobile for constructing the forcesensitive response boxes and Christopher Stockbridge for programming the custom computer code for interfacing the responses boxes with MATLAB. 
Open Practices Statement The data and analysis scripts for this experiment are available at https://osf.io/x6fgn/. This experiment was motivated by prior research but not preregistered.

\section{References}

Behrens, T. E., Woolrich, M. W., Walton, M. E., \& Rushworth, M. F. (2007). Learning the value of information in an uncertain world. Nat Neurosci, 10(9), 1214-1221.

Botvinick, M. M., Braver, T. S., Barch, D. M., Carter, C. S., \& Cohen, J. D. (2001). Conflict monitoring and cognitive control. Psychol Rev, $108(3), 624-652$.

Brainard, D. H. (1997). The Psychophysics Toolbox. Spat Vis, 10(4), 433436.

Egner, T. (2014). Creatures of habit (and control): a multi-level learning perspective on the modulation of congruency effects. Front Psychol, $5,1247$.

Faul, F., Erdfelder, E., Lang, A. G., \& Buchner, A. (2007). G*Power 3: a flexible statistical power analysis program for the social, behavioral, and biomedical sciences. Behav Res Methods, 39(2), 175-191.

Grant, L. D., \& Weissman, D. H. (2019). Turning distractors into targets increases the congruency sequence effect. Acta Psychol (Amst), 192, $31-41$.

Gratton, G., Coles, M. G. H., \& Donchin, E. (1992). Optimizing the use of information: strategic control of activation and responses. JEP: General, 4, 480-506.

Hazeltine, E., Lightman, E., Schwarb, H., \& Schumacher, E. H. (2011). The boundaries of sequential modulations: evidence for set-level control. J Exp Psychol Hum Percept Perform, 37(6), 1898-1914.

Hommel, B., Proctor, R. W., \& Vu, K. P. (2004). A feature-integration account of sequential effects in the Simon task. Psychol Res, 68(1), $1-17$.

Huettel, S. A., Mack, P. B., \& McCarthy, G. (2002). Perceiving patterns in random series: dynamic processing of sequence in prefrontal cortex. Nat Neurosci, 5(5), 485-490.
Logan, G. D., \& Bundesen, C. (2003). Clever homunculus: is there an endogenous act of control in the explicit task-cuing procedure? $J$ Exp Psychol Hum Percept Perform, 29(3), 575-599.

Mattler, U. (2005). Flanker effects on motor output and the late-level response activation hypothesis. $Q J$ Exp Psychol A, 58(4), 577-601.

Miller, E. K., \& Cohen, J. D. (2001). An integrative theory of prefrontal cortex function. Annual Review of Neuroscience, 24, 167-202.

Miller, J., \& Rouast, N. M. (2016). Dissociations of spatial congruence effects across response measures: an examination of delta plots. Psychol Res, 80(5), 805-820.

Ridderinkhof, K. R. (2002). Micro- and macro-adjustments of task set: activation and suppression in conflict tasks. Psychol Res, 66(4), 312323.

Schmidt, J. R., \& De Houwer, J. (2011). Now you see it, now you don't: controlling for contingencies and stimulus repetitions eliminates the Gratton effect. Acta Psychol (Amst), 138(1), 176-186.

Stürmer, B., Leuthold, H., Soetens, E., Schroter, H., \& Sommer, W. (2002). Control over location-based response activation in the Simon task: behavioral and electrophysiological evidence. $J$ Exp Psychol Hum Percept Perform, 28(6), 1345-1363.

Weissman, D. H., Colter, K. M., Grant, L. D., \& Bissett, P. G. (2017). Identifying stimuli that cue multiple responses triggers the congruency sequence effect independent of response conflict. $J$ Exp Psychol Hum Percept Perform, 43(4), 677-689.

Weissman, D. H., Egner, T., Hawks, Z., \& Link, J. (2015). The congruency sequence effect emerges when the distracter precedes the target. Acta Psychologica (Amst), 156, 8-21.

Weissman, D. H., Hawks, Z. W., \& Egner, T. (2016). Different levels of learning interact to shape the congruency sequence effect. $J$ Exp Psychol Learn Mem Cogn, 42(4), 566-583.

Publisher's note Springer Nature remains neutral with regard to jurisdictional claims in published maps and institutional affiliations. 